\title{
Les effets non-linéaires en interaction houle-structure et leur modélisation
}

\author{
BERNARD MOLIN ${ }^{\mathrm{a}}$ \\ École généraliste d’ingénieurs de Marseille, 13451 Marseille Cedex 20, France
}

Reçu le 17 décembre 2004, accepté le 13 octobre 2005

\begin{abstract}
Résumé - En première approximation, l'étude de l'action des vagues sur les structures, fixes ou flottantes, se fait dans le cadre de théories linéarisées. Les manifestations d'effets non-linéaires sont cependant nombreuses et ont diverses origines : non-linéarités mécaniques, variations de surface mouillée, effets visqueux (séparation), non-linéarités des conditions de surface libre. On ne considère ici que ce dernier type de nonlinéarité. Deux approches sont décrites, où la mise en équation est dans les deux cas basée sur la théorie potentielle. Des applications sont présentées pour deux géométries de référence : un cylindre vertical, et une plaque plane verticale, travers à la houle. La première méthode procède par approximations successives, sur la base d'un développement en série de perturbation, dont la théorie linéaire constitue le premier ordre. Un intérêt du deuxième ordre d'approximation, bien maîtrisé aujourd'hui, est de faire apparaître des efforts dans un domaine de fréquences élargi, où sont susceptibles de se trouver des fréquences propres du système étudié. La complexité du problème de diffraction au troisième ordre a dissuadé la plupart des chercheurs de s'y aventurer. On avance ici que les interactions tertiaires entre houle incidente et houle réfléchie par la structure peuvent jouer un rôle très important, méconnu jusqu'à récemment, en particulier sur les phénomènes de « run-up » et d'envahissement. La deuxième approche consiste à résoudre, en temps et en espace, les équations initiales du problème en tenant compte exactement des conditions non-linéaires de surface libre. On aboutit ainsi à des équivalents numériques des bassins à houle physiques. On les décrit sommairement et on présente quelques applications.
\end{abstract}

Mots clés : Interaction houle-structure / hydrodynamique / non-linéarité

\begin{abstract}
Nonlinear phenomena in wave-body interaction: description and theoretical modeling. At first approximation, the study of wave interaction with fixed or floating bodies is carried out within a linear frame. However nonlinear effects are numerous and they have diverse origins: mechanical nonlinearities, variation in time of the wetted part of the hull, viscous phenomena (flow separation), nonlinear free surface equations. We focus here on the latter type of nonlinearities. Two different approaches are described, both being based on potential flow theory. Practical applications are given for two basic geometries: a vertical cylinder and a vertical plate, perpendicular to the wave direction. In the first approach, one proceeds through successive approximations, based on a perturbation series development. The first-order of approximation coincides with the linear theory. The main interest of the second-order of approximation, well mastered nowadays, is that it yields excitation loads in an enlarged frequency domain, encompassing most of the natural frequencies of the system considered. At third-order the complexity of the equations becomes dissuasive and few researchers have ventured there. We suggest that third-order (or tertiary) interactions, between incoming waves and reflected waves by the structure, can play a very important role, overlooked so far, in phenomena such as run-up or green water. In the second approach one integrates in time and space the nonlinear equations of the initial boundary value problem, with the free surface equations being exactly satisfied. In this way one obtains numerical equivalents of the physical wave-tanks. They are briefly described and some illustrative results are given.
\end{abstract}

Key words: Wave-body interaction / hydrodynamics / nonlinear

a Auteur correspondant : bmolin@egim-mrs.fr 


\section{Introduction}

On s'intéresse ici au comportement sur houle de structures telles que celles rencontrées en offshore pétrolier, loin de la côte, en profondeur quasi constante, et au point fixe. On élimine donc d'entrée tous les problèmes, rencontrés en génie côtier, de propagation de la houle du large à la côte, de son interaction avec la bathymétrie et avec le trait de côte, etc. On élimine aussi l'effet de la vitesse d'avance, propre à la conception navale.

Ces structures pétrolières sont extrêmement variées : massives (barges, plates-formes gravitaires) ou ajourées (treillis métalliques), fixes ou flottantes, voire déformables.

L'étude de leur comportement à la houle se fait d'abord dans le cadre d'une théorie linéaire. L'état de mer, supposé stationnaire, étant défini par sa densité spectrale d'énergie $S(\omega, \beta)$ où $\omega$ est la pulsation et $\beta$ la direction de propagation, on détermine la fonction de transfert complexe $f_{X}(\omega, \beta)$ de la quantité $X$ étudiée (mouvement, effort, contrainte). La densité spectrale de $X$ s'écrit alors :

$$
S_{X}(\omega)=\int_{0}^{2 \pi} S(\omega, \beta) f_{X}(\omega, \beta) f_{X}^{*}(\omega, \beta) \mathrm{d} \beta
$$

On suppose de plus, habituellement, que la houle est un processus gaussien, ce qui est implicitement lié à la légitimité d'écrire l'élévation de surface libre sous la forme :

$$
\eta(x, y, t)=\sum_{i=1}^{\infty} A_{i} \cos \left(k_{i} x \cos \beta_{i}+k_{i} y \sin \beta_{i}-\omega_{i} t+\theta_{i}\right)
$$

où les amplitudes élémentaires $A_{i}$ se relient au spectre d'énergie $S(\omega, \beta)$ de la houle par $A_{i}^{2}=2 S\left(\omega_{i}, \beta_{i}\right) \delta \omega_{i} \delta \beta_{i}$ et où les déphasages $\theta_{i}$ sont aléatoires et indépendants. Il s'ensuit que la réponse $X$ est également un processus gaussien, le plus souvent à bande étroite : ses maxima suivent alors une loi de Rayleigh, d'où l'on déduit facilement valeurs de design ou endommagement par fatigue.

A contrario on peut distinguer deux types de comportement non-linéaires :

- la réponse prend place aux mêmes fréquences que l'entrée (la houle) mais ne lui est pas strictement proportionnelle. En d'autres termes la fonction de transfert $f_{X}$ présente une certaine sensibilité à l'état de mer, et le processus $X$ n'est pas gaussien;

- la réponse prend place à des pulsations $\Omega_{j}$ complètement différentes de celles $\omega_{i}$ de l'entrée, pour lesquelles l'énergie associée $S\left(\Omega_{j}, \beta\right)$ est nulle.

Un exemple du premier type de comportement nonlinéaire est le roulis des navires et des barges à la résonance. Typiquement les périodes propres en roulis sont comprises entre 10 et 20 secondes, ce sont des périodes où le contenu énergétique de la houle est important. Le caractère non-linéaire de la réponse provient alors

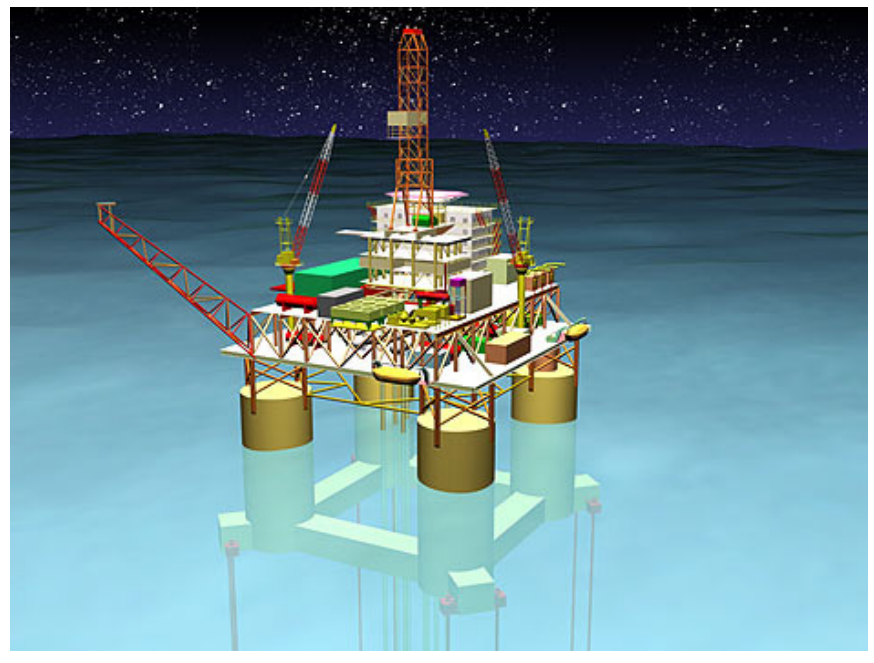

Fig. 1. Plate-forme sur lignes tendues du champ de Magnolia, golfe du Mexique (source www.offshore-technology.com).

de ce que, parmi les mécanismes dissipateurs d'énergie, le décollement de la couche limite aux bouchains joue un rôle majeur. Le couple amortisseur qui en résulte se relie alors au carré de la vitesse angulaire (plus exactement à $\dot{\alpha}|\dot{\alpha}|$ où $\alpha$ est l'angle de roulis), l'excitation étant, elle, assez bien prédite par la théorie linéaire.

Les périodes de houle couvrent un domaine assez étroit, allant de quelques secondes à environ 20 secondes. Les systèmes offshore sont souvent conçus de telle manière que les périodes propres de leurs mouvements se situent en dehors de cet intervalle. C'est ainsi que les ancrages, souples, confèrent aux mouvements horizontaux des structures flottantes des périodes propres habituellement bien supérieures à la minute. Pour autant des réponses résonnantes sont observées, entretenues par des efforts d'origine non-linéaire, prenant place aux différences deux à deux des pulsations du spectre de houle. Pour grande partie ces efforts proviennent des variations de surface mouillée de la carène : l'intégration d'une pression fluctuante sur une surface également fluctuante donne des résidus d'ordre deux dont une partie varie en temps suivant le signal enveloppe à la houle : bien que petits, en magnitude, devant ceux de premier ordre, ces efforts de deuxième ordre à basse fréquence sont à l'origine des comportements dits de dérive lente.

À l'inverse, les ancrages verticaux des plates-formes sur lignes tendues (Fig. 1) sont extrêmement raides, leur conférant des périodes propres en roulis, tangage et pilonnement bien en dessous des périodes de houle, typiquement de l'ordre de 3 secondes. Pour autant ces mouvements ne sont pas complètement éliminés et des réponses résonnantes, dites de «springing » et « ringing », sont susceptibles de se produire. Elles ont fait l'objet d'intenses recherches en hydrodynamique et il a été établi que leur origine est clairement liée aux non-linéarités des conditions de surface libre. 
On l'entrevoit, les effets non-linéaires qui prennent place dans l'interaction houle structure sont d'origines fort diverses : certaines sont liées au caractère visqueux du fluide et aux forces de traînée qui en résultent, d'autres aux variations de surface mouillée de la carène, au comportement mécanique de la structure, etc. Il n'est pas envisageable, dans le cadre de cette communication, de les couvrir de manière exhaustive. On a donc choisi de la consacrer au type de non-linéarité qui semble être la plus spécifique de l'hydrodynamique, celle associée aux conditions de surface libre.

\section{2 Équations générales}

Les structures offshore sont classiquement divisées en « petits corps » et en « grands corps », suivant la dimension de leurs éléments constitutifs vis-à-vis de l'amplitude des vagues.

Un bon exemple de petit corps est le « jacket », consistant en un assemblage de barres dont le diamètre est l'ordre du mètre. Dès lors que l'amplitude du mouvement des particules fluides excède cette valeur, l'écoulement se sépare : du point de vue de la modélisation, le choix doit se faire entre méthodes empiriques (la formule de Morison) ou résolution des équations de Navier-Stokes.

On ne s'intéresse ici qu'aux grands corps, donc tels que les effets visqueux soient négligeables, au moins en première approximation. Il est alors légitime de faire appel aux hypothèses de fluide parfait (et incompressible) et d'écoulement irrotationnel, et donc au formidable outil qu'est la théorie potentielle. On rappelle que la vitesse fluide, soit $\vec{V}$, est le gradient du potentiel $\Phi$, et que la pression $p$ s'y relie par la relation de Bernoulli-Lagrange :

$$
p=p_{0}-\rho g z-\rho \Phi_{t}-\frac{1}{2} \rho(\nabla \Phi)^{2}
$$

où la pression de référence $p_{0}$ est la pression atmosphérique.

On utilise ici un repère galiléen $O x y z$ tel que $O x y$ coïncide avec le plan de surface libre au repos et que l'axe $O z$ soit ascendant.

Le problème à résoudre se pose alors de la manière suivante :

Soit $\Phi(x, y, z, t)$ le potentiel des vitesses de l'écoulement, scindé en deux composantes :

$$
\Phi=\Phi_{\mathrm{I}}+\Phi_{\mathrm{P}}
$$

- $\Phi_{\mathrm{I}}$ est le potentiel des vitesses de la houle incidente, en l'absence de la structure. On suppose en général que cette houle se propage sur un océan infini de profondeur constante $(h)$.

- $\Phi_{\mathrm{P}}$ est le potentiel dit de perturbation.
Le problème aux limites vérifié par le potentiel $\Phi$ s'écrit :

$$
\begin{aligned}
& \Delta \Phi=0 \quad \text { dans le domaine fluide } \\
& \frac{\mathrm{d} S}{\mathrm{~d} t}=\nabla \Phi \cdot \nabla S+S_{t}=0 \quad \text { à la surface libre } \\
& S(x, y, z, t)=0 \\
& g z+\Phi_{t}+\frac{1}{2}(\nabla \Phi)^{2}=0 \text { à la surface libre } \\
& S(x, y, z, t)=0 \\
& \text { (condition dynamique) } \\
& \Phi_{z}=0 \quad \text { sur le fond marin } \\
& z=-h \\
& \nabla \Phi \cdot \vec{n}=\vec{U} \cdot \vec{n} \quad \text { sur la carène } S_{C} \\
& \Phi \simeq \Phi_{I} \quad x^{2}+y^{2}=R^{2} \rightarrow \infty
\end{aligned}
$$

- La condition de Laplace (5) exprime la conservation de la masse $(\operatorname{div} \vec{V}=0)$.

- La condition « cinématique » (6) exprime qu'une particule à la surface libre y reste.

- La condition «dynamique $»(7)$ exprime que la pression à la surface libre (côté fluide) est égale à la pression atmosphérique. On néglige ainsi la tension superficielle, sans effet dès lors que les longueurs d'onde dépassent une dizaine de centimètres.

- Les conditions (8) et (9) expriment le glissement du fluide sur le fond marin et sur la surface mouillée de la structure, dont $\vec{U}$ est la vitesse locale et $\vec{n}$ le vecteur normal.

- Enfin on exprime (10) qu'on retrouve, à l'infini, la houle incidente, où les perturbations apportées par la structure disparaissent.

Pour résoudre un tel problème, il faut évidemment commencer par spécifier la houle incidente, dont le potentiel $\Phi_{\mathrm{I}}$ vérifie également les conditions (5-8) (mais avec une surface libre différente $\left.S_{\mathrm{I}}(x, y, z, t)=0\right)$. Visuellement, ce peut être une houle régulière (périodique) ou irrégulière, mono- ou multi-directionnelle.

Il faut aussi, si la structure est flottante et libre de répondre à la houle, coupler au système (5-10) les équations décrivant son mouvement (ou ses déformations s'il s'agit d'une structure déformable). On va ici supposer que la structure est fixe et indéformable $(\vec{U} \equiv 0)$. Malgré cette (petite) simplification, la résolution du problème aux limites (5-10) pose d'énormes difficultés :

1. le domaine fluide est illimité ;

2. sa frontière supérieure, la surface libre $S(x, y, z, t)=0$ est une inconnue du problème;

3. les conditions (6) et (7) qui y sont affichées sont nonlinéaires.

On peut remarquer, néanmoins, que les autres équations du problème (l'équation de Laplace et la condition de glissement sur le fond et sur la carène immobile) sont, elles, linéaires. Les non-linéarités du problème sont donc associées uniquement aux conditions de surface 
libre. (Comme on l'a déjà écrit, d'autres non-linéarités s'introduisent lorsqu'on passe au calcul des efforts, la pression (3) contenant un terme quadratique et étant intégrée sur une surface fluctuante.)

Une technique de résolution, due à Stokes, consiste à supposer que le problème est faiblement non-linéaire et à rechercher la solution sous la forme d'un développement en série d'un petit paramètre $\epsilon$, qui s'identifie à la cambrure de la houle :

$$
\begin{aligned}
\Phi(x, y, z, t)=\Phi^{(0)}(x, y, z)+ & \epsilon \Phi^{(1)}(x, y, z, t) \\
& +\epsilon^{2} \Phi^{(2)}(x, y, z, t)+\ldots
\end{aligned}
$$

en même temps que des développements de Taylor permettent de «travailler» dans le domaine fluide au repos $-h \leq z \leq 0$ :

$$
\begin{array}{r}
\Phi(x, y, \eta, t)=\Phi(x, y, 0, t)+\eta(x, y, t) \Phi_{z}(x, y, 0, t) \\
+\frac{1}{2} \eta^{2}(x, y, t) \Phi_{z z}(x, y, 0, t)+\ldots
\end{array}
$$

la surface libre étant alors paramétrée sous la forme $z=$ $\eta(x, y, t)=\eta^{(0)}+\epsilon \eta^{(1)}+\epsilon^{2} \eta^{(2)}+\ldots$

Le terme d'ordre zéro $\Phi^{(0)}$ correspond à l'écoulement en l'absence de houle, qui peut être celui associé à un courant ou une vitesse d'avance. Leur prise en compte peut inciter à introduire un deuxième petit paramètre, le nombre de Brard $\tau=U_{C} \omega_{e} / g$, où $U_{C}$ est la vitesse du courant (d'avance) et $\omega_{e}$ la pulsation dite de rencontre. On suppose ici que $\Phi^{(0)} \equiv \eta^{(0)} \equiv 0$.

Stokes, dans les années 1840, a appliqué cette technique au cas de la houle régulière (sans obstacle) et poussé le développement (11) à l'ordre 5. Il a fallu attendre une centaine d'années pour voir la résolution du problème linéarisé (l'ordre 1) de diffraction d'une houle régulière par un cylindre vertical [1]. On rend compte dans la section 3 des développements récents et en cours sur la résolution du problème de diffraction aux ordres suivants.

Une seconde technique, très récente, consiste à intégrer numériquement en temps et en espace les équations (5-10), en y couplant, éventuellement, celles décrivant le comportement mécanique de la structure étudiée. On aboutit ainsi à des « bassins à houle numériques », équivalents informatiques des bassins à houle physiques. Cette approche est décrite dans la section 4.

\section{Méthodes asymptotiques}

\subsection{Premier ordre d'approximation}

À l'ordre un en $\epsilon$, les conditions de surface libre (6) et (7) se réduisent à la simple condition

$$
g \Phi_{z}^{(1)}(x, y, 0, t)+\Phi_{t t}^{(1)}(x, y, 0, t)=0
$$

l'élévation de surface libre se déduisant du potentiel par :

$$
\eta^{(1)}(x, y, t)=-\frac{1}{g} \Phi_{t}^{(1)}(x, y, 0, t)
$$

Le problème étant linéaire, il suffit de le résoudre dans le cas particulier d'une houle régulière, de potentiel incident :

$$
\begin{aligned}
\Phi_{I}^{(1)}(x, z, t) & =\frac{A g}{\omega} \frac{\cosh k(z+h)}{\cosh k h} \sin (k x-\omega t) \\
& =\Re\left\{\varphi_{I}^{(1)}(x, z) \mathrm{e}^{-\mathrm{i} \omega t}\right\}
\end{aligned}
$$

et d'élévation de surface libre :

$$
\eta^{(1)}(x, t)=A \cos (k x-\omega t)
$$

qui est donc une simple sinusoïde d'amplitude $A$ et de longueur d'onde $2 \pi / k$, le nombre d'onde $k$ étant relié à la pulsation $\omega$ par la relation de dispersion (déduite de (13)) :

$$
\omega^{2}=g k \tanh k h
$$

Lorsque le problème est résolu, comme ici, dans le domaine fréquentiel, la condition de disparition à l'infini du potentiel de perturbation $\Phi_{P}^{(1)}=\Re\left\{\varphi_{P}^{(1)} \exp (-\mathrm{i} \omega t)\right\}$ ne suffit pas à assurer l'unicité de la solution. Il faut en effet éliminer des solutions non physiques, ayant la forme de clapotis. La condition de « radiation » (ou « rayonnement ») est souvent écrite sous la forme dite de Sommerfeld :

$$
\lim _{R \rightarrow \infty} \sqrt{R}\left(\frac{\partial \varphi_{P}^{(1)}}{\partial R}-\mathrm{i} k \varphi_{P}^{(1)}\right)=0
$$

exprimant donc que le champ de vagues perturbé est asymptotiquement progressif dans la direction radiale.

Dans le cas d'un cylindre vertical, de rayon $a$, le problème de diffraction a pour solution, en coordonnées cylindriques $(R, \theta, z)$ :

$\varphi_{P}^{(1)}=\mathrm{i} \frac{A g}{\omega} \frac{\cosh k(z+h)}{\cosh k h} \sum_{m=-\infty}^{\infty} \mathrm{i}^{m} \frac{J_{m}^{\prime}(k a)}{H_{m}^{\prime}(k a)} H_{m}(k R) \mathrm{e}^{\mathrm{i} m \theta}$

où $J_{m}$ est la fonction de Bessel de première espèce d'ordre $m$ et $H_{m}$ la fonction de Hankel : $H_{m}=J_{m}+\mathrm{i} Y_{m}$. Cette solution a été obtenue en 1940 par Havelock [1] dans le cas particulier de la profondeur infinie et étendue par Mc Camy et Fuchs [2] à la profondeur finie en 1954.

Dans le cas général d'une structure de forme quelconque, la résolution fait appel à un modèle numérique. La technique la plus utilisée est celle de l'équation intégrale, le potentiel $\varphi_{P}^{(1)}$ étant par exemple recherché sous la forme d'une distribution de sources, soit $\sigma$, sur la carène :

$\varphi_{P}^{(1)}(x, y, z)=\frac{1}{4 \pi} \iint_{S_{C}} \sigma(\xi, \eta, \zeta) G(x, y, z ; \xi, \eta, \zeta) \mathrm{d} S_{\xi \eta \zeta}$

la fonction de Green $G$ vérifiant l'équation de Laplace, les conditions de surface libre et de glissement sur le fond, et de « rayonnement» (ou radiation) à l'infini. La satisfaction de la condition de glissement sur la carène se traduit alors par une équation intégrale, résolue numériquement. De nombreux modèles, passés dans le domaine industriel, 
sont devenus, depuis une vingtaine d'années, des outils standard de conception des structures offshore. Ils fournissent les fonctions de transfert $f_{X}(\omega, \beta)$ de toutes les grandeurs utiles au concepteur pour leur dimensionnement (mouvements, contraintes, surélévations de surface libre le long de la carène, pressions locales, etc.).

\subsection{Deuxième ordre d'approximation}

On passe ici au terme $\Phi^{(2)}$ du développement (11). Il obéit à la condition de surface libre

$\Phi_{t t}^{(2)}+g \Phi_{z}^{(2)}=-\eta^{(1)} \frac{\partial}{\partial z}\left(\Phi_{t t}^{(1)}+g \Phi_{z}^{(1)}\right)-2 \nabla \Phi^{(1)} \cdot \nabla \Phi_{t}^{(1)}$

toujours affichée en $z=0$. Le second membre est une forme quadratique de la solution à l'ordre un. Cela entraîne que, si la houle incidente est régulière de pulsation $\omega$, le potentiel de deuxième ordre apparaît aux pulsations $2 \omega$ et 0 . Dans le cas d'une houle irrégulière de pulsations porteuses $\omega_{i}$, il prend place aux pulsations $\omega_{i} \pm \omega_{j}$. Il en va de même pour les efforts de deuxième ordre qui, par rapport aux efforts d'ordre un, couvrent donc un domaine de fréquences bien plus étendu, où sont susceptibles de se trouver des fréquences critiques des systèmes étudiés. C'est le cas du mouvement horizontal des structures ancrées, mouvement dit de dérive lente, dont les périodes propres sont de l'ordre de la minute, bien audelà des périodes de houle : les efforts de deuxième ordre à basse fréquence (en mode différence $\omega_{i}-\omega_{j}$ ) en sont à l'origine. C'est aussi le cas des comportements dits de « springing » des plates-formes sur lignes tendues, à haute fréquence, dus aux efforts de deuxième ordre de mode somme $\left(\omega_{i}+\omega_{j}\right)$.

À nouveau on sépare terme incident $\Phi_{I}^{(2)}$ (pour lequel la résolution est aisée) et terme de perturbation $\Phi_{P}^{(2)}$. On se place dans le cas d'une houle régulière où $\Phi_{P}^{(2)}$ se décompose en un terme indépendant du temps, sans grand intérêt, et un terme fluctuant à la pulsation double :

$$
\Phi_{P}^{(2)}(x, y, z, t)=\varphi_{P 0}^{(2)}(x, y, z)+\Re\left\{\varphi_{P}^{(2)}(x, y, z) \mathrm{e}^{-2 \mathrm{i} \omega t}\right\}
$$

$\varphi_{P}^{(2)}(x, y, z)$ est solution du problème aux limites :

$$
\Delta \varphi_{P}^{(2)}=0 \quad \text { dans le domaine fluide }
$$

$$
\begin{aligned}
& g \varphi_{P z}^{(2)}-4 \omega^{2} \varphi_{P}^{(2)}=-\frac{\mathrm{i} \omega}{2 g} \varphi^{(1)}\left(g \varphi_{z z}^{(1)}-\omega^{2} \varphi_{z}^{(1)}\right) \\
&+\mathrm{i} \omega \nabla \varphi^{(1)^{2}}+\frac{\mathrm{i} \omega}{2 g} \varphi_{I}^{(1)}\left(g \varphi_{I z z}^{(1)}\right. \\
&\left.-\omega^{2} \varphi_{I z}^{(1)}\right)-\mathrm{i} \omega \nabla \varphi_{I}^{(1)^{2}} \\
& \text { en } z=0
\end{aligned}
$$

$\varphi_{P z}^{(2)}=0 \quad$ sur le fond marin $z=-h$ $\nabla \varphi_{P}^{(2)} \cdot \vec{n}=-\nabla \varphi_{I}^{(2)} \cdot \vec{n} \quad$ sur la carène $S_{C}$

$$
\operatorname{Rad}\left\{\varphi_{P}^{(2)}\right\} \quad R \rightarrow \infty
$$

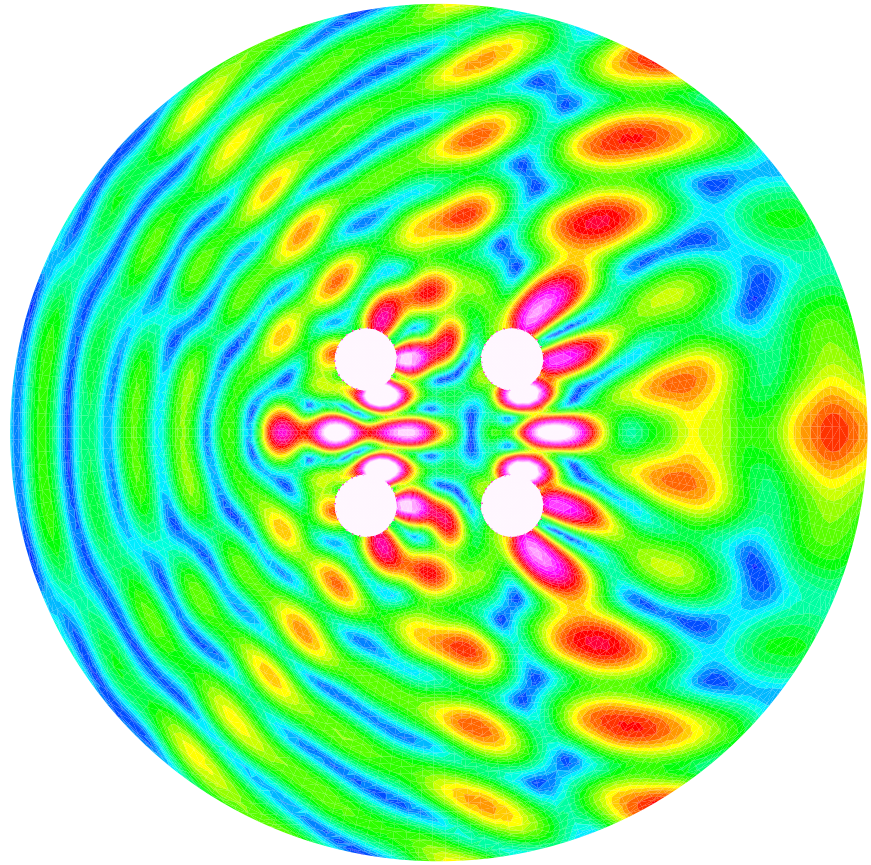

Fig. 2. Diffraction d'une houle régulière sur un ensemble de quatre cylindres verticaux. Module de la composante de deuxième ordre de l'élévation de surface libre [3].

Par rapport à l'ordre un, la complication provient du caractère non homogène de la condition de surface libre (24) dont le second membre décroît lentement avec la distance radiale $R$ (en $R^{-1 / 2}$ ). Physiquement il peut s'interpréter comme une pression dynamique agissant sur tout le plan de surface libre. Il en résulte que le champ de vagues associé à $\varphi_{P}^{(2)}$ n'est pas asymptotiquement radial à l'infini, au contraire de celui du premier ordre : il se décompose en ondes « libres », vérifiant asymptotiquement la condition homogène de surface libre, et ondes « liées » au système d'onde de premier ordre. Une illustration est fournie par la figure 2, [3].

Ce n'est que relativement récemment que ces difficultés ont été bien identifiées et résolues [4]. Des solutions semi-analytiques ont été obtenues pour des géométries simples comme le cylindre vertical (voir, par exemple [5]). Une caractéristique remarquable du potentiel de perturbation de deuxième ordre, en mode somme, est qu'il pénètre profondément la colonne d'eau lorsque les pulsations $\omega_{i}$ et $\omega_{j}$ sont voisines (d'où les problèmes de résonance rencontrés avec les plates-formes sur lignes tendues, en dépit de leurs forts tirants d'eau).

Il existe maintenant quelques modèles numériques, passés au stade industriel, qui permettent le calcul des « fonctions de transfert quadratiques » (QTF) des efforts de deuxième ordre en mode somme et mode différence, pour tous les couples $\left(\omega_{i}, \omega_{j}\right)$ des pulsations présentes dans le signal houle. Ce sont là des calculs lourds, guère envisageables au stade de pré-études.

Il est important de noter que le deuxième ordre ne remet pas en cause le premier ordre : au fondamental il 
rajoute des harmoniques. Il en va différemment à l'ordre suivant d'approximation où l'ordre un est remis en cause.

\subsection{Troisième ordre d'approximation}

On passe donc au terme $\Phi^{(3)}$ du développement (11), qui vérifie la condition de surface libre :

$$
\begin{aligned}
\Phi_{t t}^{(3)}+g \Phi_{z}^{(3)}= & -\eta^{(2)} \frac{\partial}{\partial z}\left(\Phi_{t t}^{(1)}+g \Phi_{z}^{(1)}\right) \\
& -\eta^{(1)} \frac{\partial}{\partial z}\left(\Phi_{t t}^{(2)}+g \Phi_{z}^{(2)}+\frac{\partial}{\partial t} \nabla \Phi^{(1)^{2}}\right) \\
& -\Phi_{x}^{(1)^{2}} \Phi_{x x}^{(1)}-\Phi_{y}^{(1)^{2}} \Phi_{y y}^{(1)}-\Phi_{z}^{(1)^{2}} \Phi_{z z}^{(1)} \\
& -2 \Phi_{x}^{(1)} \Phi_{y}^{(1)} \Phi_{x y}^{(1)}-2 \Phi_{x}^{(1)} \Phi_{z}^{(1)} \Phi_{x z}^{(1)} \\
& -2 \Phi_{y}^{(1)} \Phi_{z}^{(1)} \Phi_{y z}^{(1)}-2 \frac{\partial}{\partial t}\left(\nabla \Phi^{(1)} \cdot \nabla \Phi^{(2)}\right)
\end{aligned}
$$

assez compliquée, qui est une forme cubique du potentiel de premier ordre. On en déduit donc que, si la houle incidente est régulière de pulsation $\omega$, le potentiel de troisième ordre apparaît aux pulsations $3 \omega$ et $\omega$ : on retrouve le fondamental. De même, en houle irrégulière, $\omega_{i} \pm \omega_{j} \mp \omega_{j}$ redonne $\omega_{i}$.

L'intérêt de la profession offshore pour le terme de pulsation $3 \omega$ est apparu au début des années 1990 à la suite d'essais en bassin sur un nouveau concept de plateforme sur lignes tendues, celle du champ norvégien de Heidrun, et d'autres essais réalisés sur la plate-forme gravitaire de Draugen. Dans les deux cas des comportements résonnants (baptisés « ringing ») sont apparus, prenant place à des fréquences trois ou quatre fois plus grandes que celles du pic du spectre de houle, et suggérant donc des non-linéarités du même ordre. De nombreux programmes de recherche furent alors lancés sur ce sujet, de nature expérimentale, théorique et numérique. Le calcul exact des efforts de diffraction de troisième ordre a été mené à bien par Malenica dans le cas d'un cylindre vertical soumis à une houle régulière [6]. D'autres chercheurs [7] ont abordé le cas de structures axisymétriques, à axe de révolution vertical. Ce sont là des solutions de référence, d'application pratique limitée, mais utiles pour valider des méthodes approchées (dont aucune, en fin de compte, ne s'est révélée satisfaisante, car incapable de rendre compte du champ de vagues qui apparaît à la pulsation triple).

En ce qui concerne le terme de pulsation $\omega$ que l'on récupère au second membre de l'équation de surface libre, son effet sur la houle incidente est bien connu : il oblige à modifier la relation de dispersion (17) qui, toujours pour une houle régulière et dans le cas particulier de la profondeur infinie, devient :

$$
\omega^{2}=g k\left(1+k^{2} A^{2}\right)
$$

À nombre d'onde donné, la pulsation augmente ; inversement, à pulsation imposée (par le mouvement du batteur d'un bassin d'essais par exemple), le nombre d'onde diminue et la longueur d'onde augmente.
Dans le cas de la superposition de deux ondes planes, on sait également depuis longtemps [8] que leurs interactions 《tertiaires » (d'ordre trois) entraînent des modifications mutuelles de leurs longueurs d'onde. Ce n'est que très récemment (!) que l'on a réalisé [9] que, dans le cas d'une structure soumise à une houle incidente, ce type d'interaction prenait également place entre la houle incidente et la houle réfléchie. En ce qui concerne la houle incidente, les répercussions sont très similaires à celles que causerait un haut-fond placé devant la structure : la longueur d'onde diminue, les lignes de crête se déforment, l'amplitude évolue le long de celles-ci, des phénomènes de focalisation apparaissent. Les conséquences les plus visibles sont des phénomènes de « run-up », fortes surélévations de surface libre, vers le milieu de la structure, associés éventuellement à des impacts ou des envahissements d'eau; effets certainement très préoccupants pour les navires en avarie, travers à la houle, mais complètement ignorés jusqu'à aujourd'hui!

En illustration on présente sur la figure 3 des résultats de mesure et de calcul, relatifs à une plaque verticale encastrée dans un des murs du bassin BGO-First à la Seyne-sur-mer. La longueur de la plaque est de 1,2 m, des sondes à houle sont disposées sur sa longueur, à 10, 20, 40, 60, 80 et $100 \mathrm{~cm}$ du mur. Elle est soumise à des houles régulières de périodes et de cambrures $H / L$ variables, $H$ étant la crête à creux et $L$ la longueur d'onde. La figure présente les fonctions de transfert de l'élévation le long de la plaque ( $y=0$ étant l'extrémité mur), expérimentales (haut) et théoriques (bas), pour une longueur d'onde de $1,8 \mathrm{~m}$ et des cambrures $H / L$ allant de 2 à $6 \%$. On constate une très forte sensibilité des fonctions de transfert à la cambrure, avec des valeurs dépassant 4 à l'intersection de la plaque et du mur, plus de deux fois la valeur de la théorie linéaire. On note un bon accord entre les mesures et les valeurs numériques, issues d'un modèle tenant compte des interactions tertiaires entre houle incidente et houle réfléchie [9].

\section{Les bassins à houle numériques}

La technique de perturbation exposée dans la section précédente présente le défaut de ne rien pouvoir dire quant au domaine de validité des solutions qu'elle fournit, sinon qu'elles sont asymptotiquement correctes lorsque la cambrure $k A$ tend vers zéro. Il est alors nécessaire de les confronter à des résultats de mesure, au réel ou en bassin, ou à ceux fournis par des modèles numériques qui tiennent exactement compte des non-linéarités de surface libre. Il apparaît aussi nettement que, suivant cette approche, vouloir traiter l'interaction houle-structure audelà du troisième ordre d'approximation est se lancer dans une entreprise désespérée.

Avec l'augmentation des ressources informatiques, une nouvelle voie s'est ouverte qui est la résolution, en temps et en espace, du problème aux limites des équations (5), ..., (10). Les premiers développements, en deux dimensions (dans un plan vertical), sont dus à 

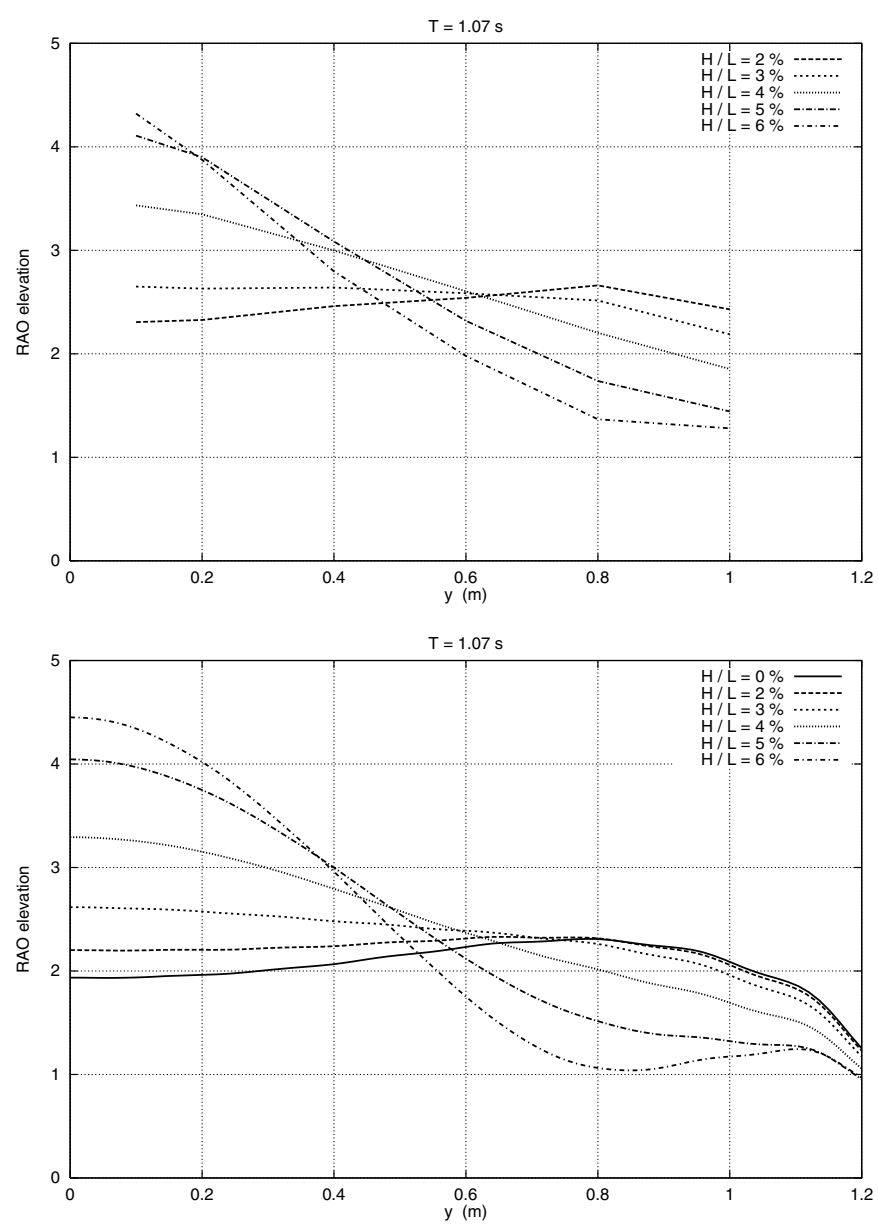

Fig. 3. Fonction de transfert de l'élévation de surface libre le long d'une demi-plaque verticale, pour différentes cambrures $H / L$. Valeurs tirées des mesures (haut) et calculs (bas).

Longuet-Higgins et Cokelet (1976) [10], avec comme application l'évolution non-linéaire et le déferlement d'un train de vagues (périodique). Ils ont combiné suivi lagrangien de la surface libre par des marqueurs et résolution du problème intérieur, à chaque pas de temps, par équation intégrale, méthode dite MEL (« Mixed Euler-Lagrange »). Les équations de surface libre s'écrivent alors simplement:

$$
\frac{\mathrm{d} \vec{X}_{i}}{\mathrm{~d} t}=\nabla \Phi_{i} \quad \frac{\mathrm{d} \Phi_{i}}{\mathrm{~d} t}=-g z_{i}+\frac{1}{2}\left(\nabla \Phi_{i}\right)^{2}
$$

Pour avancer en temps les positions $\vec{X}_{i}=\left(x_{i}, y_{i}, z_{i}\right)$ des marqueurs et les potentiels associés $\Phi_{i}$, il faut donc avoir accès aux vitesses $\nabla \Phi_{i}$. Les composantes tangentielles à la surface libre s'obtiennent par différences finies, la composante normale par résolution d'une équation intégrale. Cela suppose évidemment de travailler dans un domaine de calcul fini.

La technique de marqueurs lagrangiens permet de simuler le retournement des vagues jusqu'au déferlement où, évidemment, la simulation s'arrête. Pour aller plus loin, les approches potentielles sont inadaptées, c'est le domaine des méthodes dites VOF (« Volume Of Fluid»), SPH (« Smoothed Particle Hydrodynamics »), etc.
Une variante consiste à bloquer le mouvement horizontal des marqueurs et donc à introduire comme nouvelles variables l'élévation $\eta(x, y, t)$ et le potentiel à la surface libre $\widetilde{\Phi}(x, y, t)=\Phi(x, y, \eta(x, y, t), t)[11]$, dont la marche en temps est donnée par :

$$
\begin{aligned}
& \frac{\partial \eta}{\partial t}=w\left(1+(\nabla \eta)^{2}\right)-\nabla \widetilde{\Phi} \cdot \nabla \eta \\
& \frac{\partial \widetilde{\Phi}}{\partial t}=-g \eta+\frac{1}{2} w^{2}\left(1+(\nabla \eta)^{2}\right)-\frac{1}{2}(\nabla \widetilde{\Phi})^{2}
\end{aligned}
$$

où, comme suivant la formulation précédente, le problème est de déterminer la vitesse verticale $w$ à la surface libre pour avancer en temps. La technique d'équation intégrale continue évidemment d'être applicable. Si l'on s'intéresse à la propagation de la houle dans des domaines rectangulaires sans obstacle, d'autres méthodes, spectrales par exemple, peuvent être utilisées.

À titre d'exemple on présente brièvement le code $X W A V E$ développé par Ferrant et ses collaborateurs à l'École Centrale de Nantes [12]. XWAVE utilise les équations (31) et (32) en séparant le potentiel $\Phi$ en un terme incident $\Phi_{I}$ et un terme perturbé $\Phi_{P}$. Comme la surface libre $\eta$ de l'écoulement total peut se trouver audessus de celle $\eta_{I}$ de l'écoulement incident seul, le potentiel incident doit être prolongeable au-dessus de $\eta_{I}$. La solution retenue est de représenter $\Phi_{I}$ sous forme spectrale, par exemple celle de Rienecker et Fenton (1981) [13] si la houle incidente est régulière. L'écoulement incident peut ainsi être spécifié dans tout le domaine de calcul dès l'instant initial et le maillage peut être adapté à une bonne représentation de l'écoulement perturbé. Au-delà d'une certaine distance à la structure des termes dissipatifs sont ajoutés aux conditions de surface libre pour absorber le champ perturbé. À chaque pas de temps le problème intérieur est traité par équation intégrale, le système linéaire étant résolu par une méthode itérative. La marche en temps des équations (31) et (32) se fait par un algorithme Runge-Kutta d'ordre 4 .

La figure 4 présente, à deux instants différents, le champ de vagues obtenu dans le cas d'un cylindre vertical soumis à une houle régulière. On distingue nettement des ondes courtes, de longueur d'onde bien inférieure à celle de la houle incidente, émanant du cylindre. La figure 5 présente les composantes de pulsation $2 \omega$ et $3 \omega$ de l'effort horizontal, tirées des simulations, et comparées aux valeurs fournies par la méthode asymptotique décrite en section 3. On constate un excellent accord entre les deux séries de valeurs, qui valide mutuellement les deux approches. Ces résultats sont obtenus pour une très faible cambrure $k A=0.06$ (14\% de la cambrure dite « critique »). On peut ensuite étudier, avec le modèle $X W A V E$, le domaine de validité de l'approche asymptotique en augmentant progressivement l'amplitude de la houle incidente [12].

Une autre approche, prometteuse, s'inspire des équations de Boussinesq où le potentiel des vitesses $\Phi$ est localement exprimé sous la forme d'un développement polynomial en $z$, tronqué à un certain ordre. On relie ainsi, 

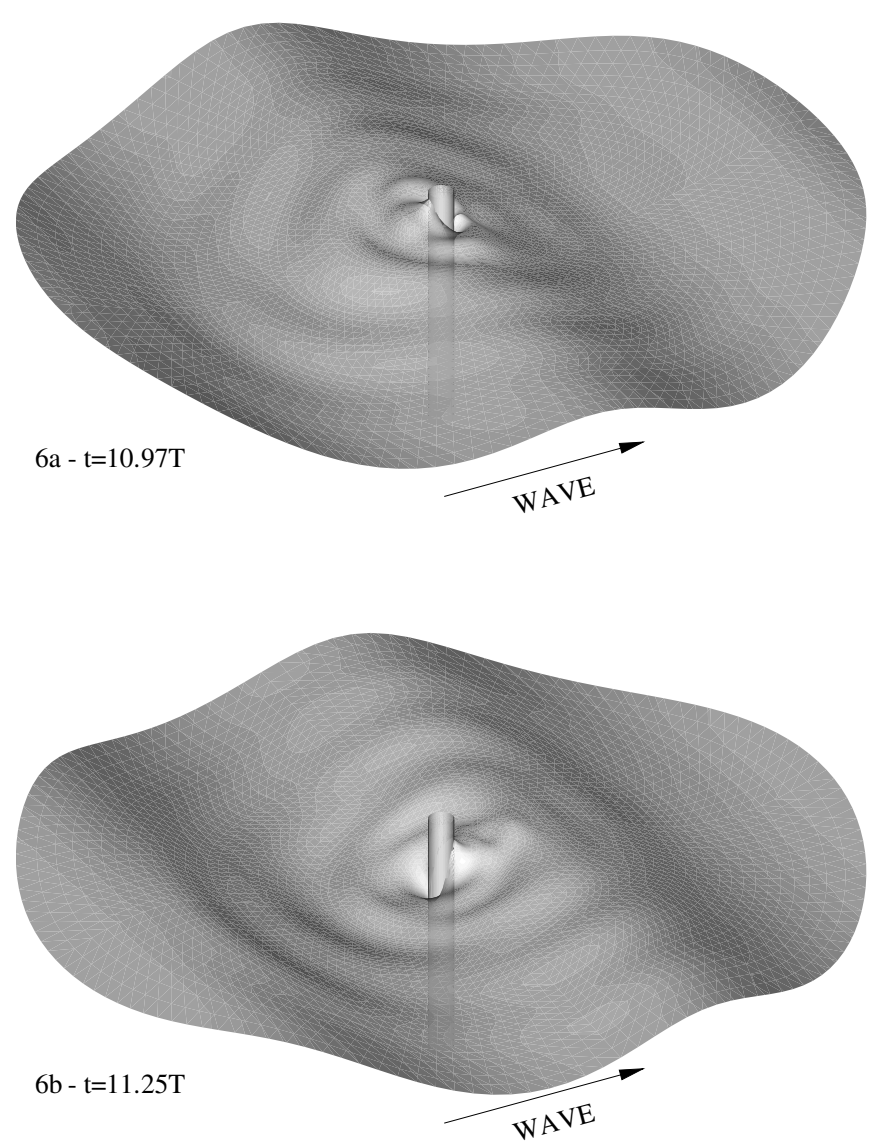

Fig. 4. Interaction non-linéaire d'une houle régulière avec un cylindre vertical. Simulation avec le programme XWAVE [12].

indirectement, la vitesse $w$ à des opérateurs différentiels du potentiel $\widetilde{\Phi}[13,14]$. Un schéma aux différences finies est ensuite appliqué. Cette méthode permet de couvrir de vastes domaines, avec une bathymétrie variable, et semble particulièrement adaptée à l'étude des phénomènes d'interactions non-linéaires en zone côtière. La figure 6 , tirée de Fuhrman (2004) [15], montre une partie du champ de vagues obtenu devant la plaque décrite à la fin de la section 3.2. La longueur d'onde est de 1,2 m. En amont de la plaque, le domaine de calcul fait 16 longueurs d'onde suivant $x$ et 10 longueurs d'onde suivant $y$, taille tout juste suffisante pour que les interactions non-linéaires entre houle incidente et houle réfléchie soient adéquatement prises en compte [9]. Les historiques d'élévation obtenus le long de la plaque sont en excellent accord avec les mesures effectuées au BGO-First.

\section{Conclusion}

On a essayé ici de donner quelque idée de l'état de la connaissance, en ce qui concerne l'effet des non-linéarités de surface libre sur l'interaction houle-structure. Bien des points n'ont pu être couverts. En particulier on ne s'est pas étendu sur la difficulté initiale que présente la spécification d'une houle incidente non-linéaire. Le
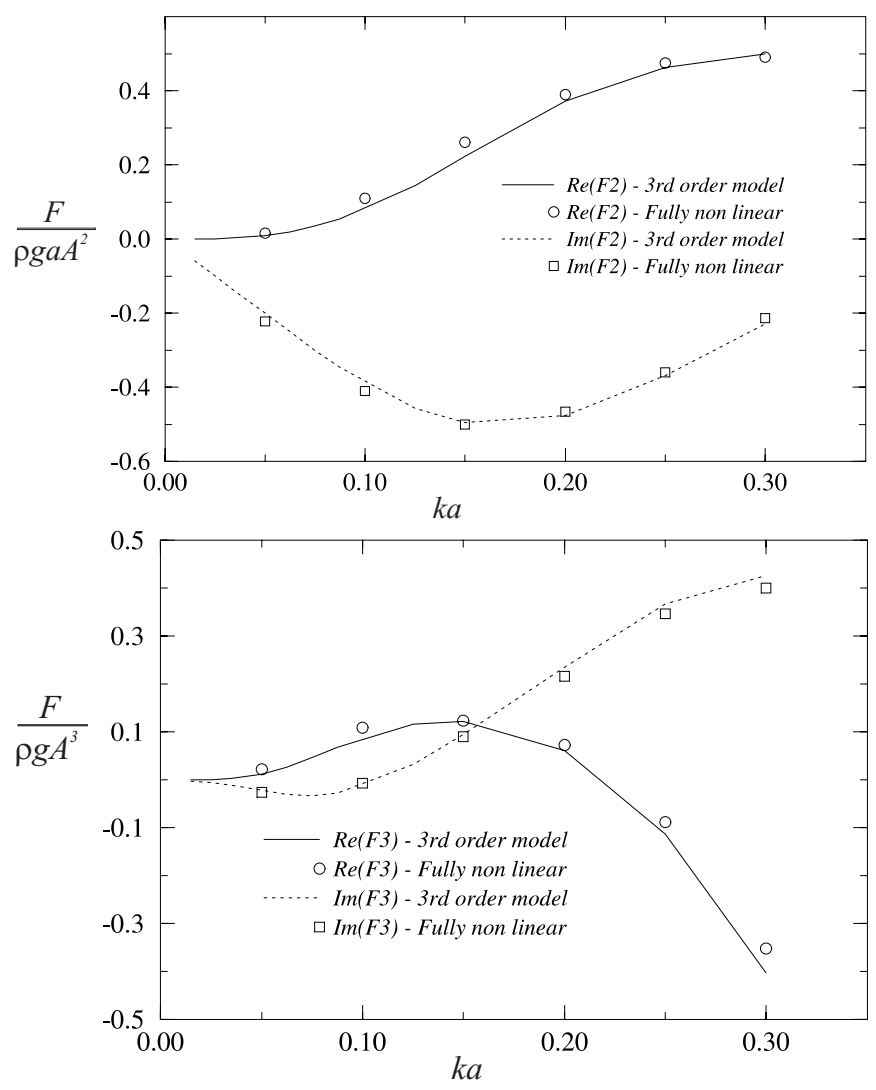

Fig. 5. Interaction non-linéaire d'une houle régulière avec un cylindre vertical. Comparaison des efforts de pulsation double (haut) et triple (bas) avec ceux fournis par la méthode asymptotique. Cambrure $k A=0.06$ [12].

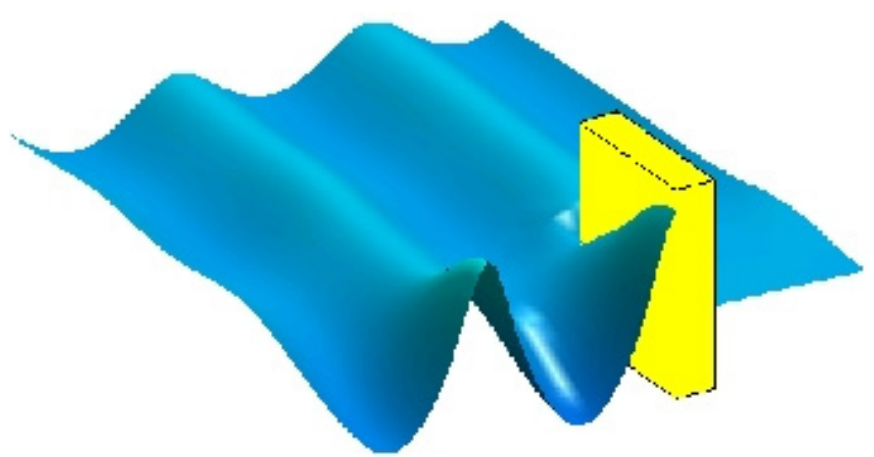

Fig. 6. Interaction non-linéaire d'une houle régulière avec une plaque verticale [15]. Longueur d'onde : 1,2 m. Cambrure $H / L: 4 \%$.

concept de houle régulière est commode d'un point de vue académique mais ne correspond pas à la réalité. La houle « irrégulière » est souvent décrite en prenant comme représentation initiale une somme de sinusoïdes telle que (2), mais dès l'ordre 2 les non-linéarités remettent en cause l'indépendance des phases et, à l'ordre 3, c'est la constance des amplitudes $A_{i}$ qui vacille : des échanges d'énergie deviennent possibles entre les différentes composantes, des phénomènes de modulation et démodulation apparaissent, entraînant éventuellement le déferlement. 
Ces interactions non-linéaires sont aussi soupçonnées de pouvoir conduire à l'apparition de vagues « anormales » (« rogue waves » ou « freak waves ») (voir, par exemple, [16-18]). De fait une importante application des bassins à houle numériques tels que décrits dans la dernière section consiste à simuler l'évolution spatiotemporelle d'états de mer en l'absence d'obstacles.

Remerciements. Les travaux de recherche décrits ici ont été accomplis, pour l'essentiel, dans le cadre de projets CLAROM (CLub pour les Actions de Recherche sur les Ouvrages en Mer; voir le site www.clarom.com).

\section{Références}

[1] T.H. Havelock, The pressure of water waves upon a fixed obstacle on water, Proc. R. Soc. London A 175 (1940) 409-421

[2] R.C. McCamy, R.A. Fuchs, Wave forces on a pile: a diffraction theory, Tech. Memo. 69 (1954) U.S. Army Corps of Engineers

[3] Y.-M. Scolan, Contribution à l'étude des non-linéarités de surface libre. Deux cas d'application : clapotis dans un bassin rectangulaire; diffraction au second ordre sur un groupe de cylindres verticaux, Thèse de doctorat, Paris VI, 1989

[4] B. Molin, Second-order diffraction loads upon threedimensional bodies, Appl. Ocean Res. 1 (1979) 197-202

[5] J.N. Newman, The second-order wave force on a vertical cylinder, J. Fluid Mech. 320 (1996) 417-443

[6] Š. Malenica, B. Molin, Third-harmonic wave diffraction by a vertical cylinder, J. Fluid Mech. 302 (1995) 203-229

[7] B. Teng, S. Kato, Third-order wave force on axisymmetric bodies, Ocean Eng. 29-7 (2002) 815-843
[8] M.S. Longuet-Higgins, O.M. Phillips, Phase velocity effects in tertiary wave interactions, J. Fluid Mech. 12 (1962) 333-336

[9] B. Molin, F. Remy, O. Kimmoun, E. Jamois, The role of tertiary wave interactions in wave-body problems, J. Fluid Mech. 528 (2005) 323-354

[10] M.S. Longuet-Higgins, E.D. Cokelet, The deformation of steep surface waves on water. 1. A numerical method of computation, Proc. R. Soc. London A 364 (1976) 1-26

[11] V.E. Zakharov, Stability of periodic waves of finite amplitude on the surface of a deep fluid, J. Appl. Mech. Tech. Phys. 9 (1968) 190-194

[12] P. Ferrant, Š. Malenica, B. Molin, Nonlinear wave loads and runup on a vertical cylinder, Nonlinear water wave interaction, O. Mahrenholtz et M. Markiewicz (ed.), WIT Press, 1999, pp. 101-135

[13] Y. Agnon, P.A. Madsen, H.A. Schäffer, A new approach to high-order Boussinesq models, J. Fluid Mech. 399 (1999) 319-333

[14] P.A. Madsen, H.B. Bingham, H. Liu, A new Boussinesq method for fully nonlinear waves from shallow to deep water, J. Fluid Mech. 462 (2002) 1-30

[15] D.R. Fuhrman, Numerical solutions of Boussinesq equations for fully nonlinear and extremely dispersive water waves, Ph.D. Thesis, Technical University of Denmark, 2004

[16] F. Dias, Ch. Kharif, Nonlinear gravity and capillarygravity waves, Annu. Rev. Fluid Mech. 31 (1999) 301-346

[17] M. Olagnon, Vagues extrêmes - Vagues scélérates, 2000, http://www.ifremer.fr/web-com/molagnon/jpo2000/

[18] Ch. Kharif, E. Pelinovsky, Physical mechanisms of the rogue wave phenomenon, Eur. J. Mech. B. Fluid. 22 (2003) 603-634

[19] M.M. Rienecker, J.D. Fenton, A Fourier approximation method for steady water waves, J. Fluid Mech. 104 (1981) 119-137

Retrouvez nos articles sur le site : www.edpsciences.org/meca 